A N N A L E S Annales de Bretagne et des Pays de l'Ouest

\title{
Le Peuple du rivage. Le littoral nord de la Bretagne au XVIIIe siècle
}

\section{Benoît Grenier}

\section{(2) OpenEdition}

Journals

\section{Édition électronique}

URL : http://journals.openedition.org/abpo/2702

DOI : $10.4000 /$ abpo.2702

ISBN : 978-2-7535-3304-2

ISSN : 2108-6443

Éditeur

Presses universitaires de Rennes

Édition imprimée

Date de publication : 30 décembre 2013

Pagination : 206-209

ISBN : 978-2-7535-3302-8

ISSN : 0399-0826

\section{Référence électronique}

Benoît Grenier, "Le Peuple du rivage. Le littoral nord de la Bretagne au XVIIIe siècle », Annales de Bretagne et des Pays de l'Ouest [En ligne], 120-4 | 2013, mis en ligne le 30 décembre 2013, consulté le 19 avril 2019. URL : http://journals.openedition.org/abpo/2702

Ce document a été généré automatiquement le 19 avril 2019.

(c) Presses universitaires de Rennes 


\title{
Le Peuple du rivage. Le littoral nord de la Bretagne au XVIIIe siècle
}

\author{
Benoît Grenier
}

\section{RÉFÉRENCE}

CHARPENTIER, Emmanuelle, Le Peuple du rivage. Le littoral nord de la Bretagne au XVIII ${ }^{e}$ siècle, Rennes, PUR, coll. « Histoire », 2013, 404 p.

1 Ce récent ouvrage de l'historienne Emmanuelle Charpentier est la version remaniée d'une thèse de doctorat soutenue à l'université de Rennes 2, en décembre 2009, sous la direction d'Annie Antoine qui en signe la préface. L'auteur présente une étude originale et agréable à lire qui devrait pouvoir satisfaire tant les historiens que le public averti et qui vise à comprendre le passé de la Bretagne, en particulier celui du littoral nord de la péninsule. À partir d'une réflexion sur la pertinence du concept de "sociétés littorales", E. Charpentier se demande si on peut les considérer comme des sociétés à part, si elles se considéraient comme telles; elle pose, en somme, la question de la présence d'une identité littorale dans cette région de la Bretagne au XVIII ${ }^{\mathrm{e}}$ siècle.

Le livre se divise en trois parties (usages du littoral ; menaces et conflits ; gens du littoral), elles-mêmes subdivisées en autant de chapitres. Des introductions et conclusions auraient été utiles à ces parties pour bien en montrer l'unité. Heureusement, chacun des chapitres forme un tout cohérent qui conduit de manière explicite vers le suivant. Le premier s'intéresse à l'exploitation de toutes les ressources pour montrer le degré de «maritimisation » des populations côtières qui pêchent, consomment et commercialisent les produits de la mer. Il y est question non seulement de la pêche et des ressources de l'estran, mais aussi de tout ce que la mer peut apporter, incluant les épaves de navires et les occasions de participer à la contrebande de tabac. Au chapitre deux, E. Charpentier délaisse l'horizon maritime pour observer les ruraux que sont aussi les gens du littoral. Contrairement à certains préjugés voulant que les Bretons du littoral ne soient «ni des ruraux ni des marins ", elle s'emploie à montrer qu'au contraire, si on tire parti de tout ce 
que donne la mer, on tire aussi parti de tout l'espace terrestre à des fins de culture. Elle documente aussi la capacité extraordinaire d'adaptation de ces populations qui font usage des ressources de la mer (dont le goémon) pour amender la terre et qui font preuve d'une grande polyvalence. Ce chapitre s'intéresse également à la question complexe des afféagements par lesquels on met en valeur des espaces considérés comme incultes. Ici, un détour par l'historiographie britannique sur la question fondamentale des enclosures aurait été utile. Le troisième chapitre étudie spécifiquement la zone entre terre et mer, l'estran, porteur d'usages spécifiques. Ce sont quelques-unes des plus belles pages de ce livre où sont abordées les ressources de cet espace (pêcheries, salines, moulins à marée), mais aussi l'espace de vie qu'il constitue (on le parcourt, on s'y délasse, on s'y promène et parfois on s'y baigne). Enfin, l'auteur se questionne sur le «spectacle de la mer ». Avec beaucoup de sensibilité, mais aussi toutes les préventions nécessaires, E. Charpentier se demande si les littoraux « regardaient la mer », si cet espace pouvait, comme de nos jours, constituer un paysage. La réponse, on s'en doute, n'est pas dans les documents...

3 Le chapitre quatre traite de la nécessité de protéger les ressources du littoral et en particulier du rôle de l'État qui affirme son autorité sur cet espace. Protection des terres (des grandes marées, de l'ensablement...), protection des ressources halieutiques (conscience environnementale avant la lettre) et gestion des ressources du littoral témoignent de la volonté de la Couronne de préserver l'estran. Au chapitre cinq, c'est de la protection des populations que nous entretient l'auteur, rappelant d'entrée de jeu que la mer est aussi associée à la mort, par les cadavres qu'elle déverse occasionnellement sur les rives, par le danger de noyade pour ceux qui fréquentent le rivage (plus que la mer elle-même d'ailleurs), par les maladies contagieuses que les navires rapportent de contrées lointaines et, surtout, par les dangers de la guerre "venue de la mer ». Ce dernier aspect est particulièrement concret pour les habitants des côtes nord de la Bretagne durant les guerres opposant la France à la Grande-Bretagne. Quant au dernier chapitre de la seconde partie, il traite de la protection des droits du littoral, s'intéressant aux pouvoirs royal et seigneuriaux qui se superposent et s'affrontent pour le contrôle côtier. Ces relations de pouvoir entre l'état et les seigneurs riverains de la mer révèlent la féodalité du rivage. Si cette seigneurie "agro-maritime» est remise en cause par l'affirmation du pouvoir royal sur le littoral, E. Charpentier explique que l'affrontement entre ces pouvoirs se solde par une forme d'accommodement réciproque.

Le septième chapitre ouvre la dernière partie de l'ouvrage et nous introduit finalement à proximité des "gens du littoral ». Après une réflexion sur les problèmes de terminologie pour désigner "ceux qui fréquentent la mer» (lesquels se désignent rarement euxmêmes), l'auteur montre bien que la pluriactivité est le lot d'à peu près tout le monde. Très rares sont ceux qui peuvent vivre exclusivement de la mer nous rappelle-t-elle. Elle se questionne aussi sur l'identité maritime de ces populations en recherchant des marqueurs possibles de celle-ci (désignations, vêtements...) et aborde les stratégies identitaires, recherchant vainement les "villages de marins" comme on retrouve des quartiers de marins à la ville. Au final, elle affirme que la sociabilité en mer ne se double pas d'une sociabilité à terre et que le référent paroissial prime sur la sociabilité de la mer. Elle conclut ce chapitre en proposant de parler d'une identité du littoral plutôt que d'une identité maritime. Au chapitre huit, l'ouvrage emprunte la piste de l'appel du large, se questionnant sur la notion de vocation maritime et sur l'influence des gens de mer sur les littoraux. En consultant les matricules des classes et les registres des mousses, mais aussi les archives judiciaires, E. Charpentier porte un regard attentif sur le tournant de 
l'« adolescence ", lorsque se dessinent les carrières maritimes, consciente cependant de la difficulté de déterminer la part de choix individuel dans ces itinéraires de vie. Quoi qu'il en soit, la mer est une réalité familière aux enfants du large, plus qu'à ceux de l'Argoat en tout cas. Suit une étude de l'embarquement proprement dit qui se penche sur la question de l'offre maritime, en termes d'emploi, ainsi que sur celle des préparatifs du départ. Cet avant-dernier chapitre se termine par la triste réalité du risque constant d'un départ sans retour. On y comprend combien l'absence des hommes constituait pour les femmes de marins un moment de doute et de solitude dans l'espoir du retour. À travers les lettres de Marie-Jacquette Pignot à son mari et le recours intelligent à l'étude de la sociologue Yvonne Guichard-Claudic sur les femmes de marins, E. Charpentier esquisse avec beaucoup de sensibilité le quotidien de ces "femmes de partis en mer", abordant des enjeux délicats comme la solitude, la fidélité (des hommes comme des femmes) et le retour (pas toujours synonyme d'heureuses retrouvailles...). Ces questions nous conduisent tout naturellement au neuvième et dernier chapitre qui aborde justement les stratégies de (sur)vie de ce peuple du littoral que l'on a l'impression de connaître de mieux en mieux au terme de ce magnifique ouvrage. Face à des salaires irréguliers et souvent une précarité économique, les gens du littoral sont inventifs et pragmatiques. Les formes d'entraide vont de la cohabitation et de la restructuration des ménages au recours au crédit. Il y est aussi question à nouveau des formes de pluriactivité, entre stratégie et nécessité. Finalement, ce dernier chapitre se conclut par la question des pratiques illégales, telle le transport de passagers clandestins, qui procurent aussi parfois un supplément appréciable.

5 Le " goût de l'archive » qui a piqué E. Charpentier est sans contredit contagieux. Attentive aux indices, elle a recours pour connaître ces gens du littoral à des sources variées qui reflètent les jeux d'échelles de l'analyse, entre le quantitatif et la microhistoire. Même s'agissant d'une thèse, la quantité de sources analysées est impressionnante. Une importance est accordée à l'enquête de François Le Masson du Parc, inspecteur général des pêches pour les provinces du nord-ouest de la France, qui parcourt le littoral nord de la Bretagne à deux reprises (1726 et 1731). À ce type de document émanant d'autorités administratives qui portent un regard « étrangeré » sur les sociétés qu'il observe, l'auteur a associé les archives judiciaires qu'elle sait faire parler pour reconstituer l'ordinaire des populations côtières. Les exemples sont trop nombreux pour les citer, mais elle fait preuve d'une grande habileté à faire surgir du passé des témoignages à première vue peu significatifs mais qui viennent pourtant enrichir considérablement son récit et notre compréhension de cette société. On peut regretter cependant le caractère très français et francophone de la bibliographie (néanmoins consistante) qui aurait pu s'enrichir de plus de références étrangères, notamment en provenance des îles britanniques où l'enjeu de l'identité littorale aurait fourni un cadre de comparaison utile. À quelques occasions, la comparaison avec les colonies françaises d'Amérique, en particulier dans le contexte de guerre du XVIII ${ }^{e}$ siècle, aurait aussi été profitable, par exemple à propos des milices gardecôtes qui ont un pendant terrestre au Canada à la même époque et qui défendent aussi un territoire convoité par les Britanniques et délimité à la fois par la mer et par de vastes forets tout aussi «effrayantes ». Ce détour sera peut-être pour une prochaine fois, la comparaison entre les sociétés bretonnes et canadiennes méritant très certainement qu'on s'y arrête plus longuement. Signalons enfin que, sans revendiquer faire une histoire du genre, E. Charpentier interroge toujours ses sources de manière à montrer que les femmes, les hommes, voire les enfants, entretiennent tous un rapport à cette vie littorale, mais que ces rapports ne sont pas sans être influencés par leur identité masculine ou 
féminine ou encore par les âges de la vie. À de nombreuses reprises, et dans la troisième partie en particulier, elle rappelle que la pêche et les activités du littoral ne sont pas que l'affaire des hommes et que, surtout, cette vie n'est pas sans impact sur les femmes, en particulier considérant les absences prolongées des " partis en mer ». Elle sait lire entre les lignes et émettre des hypothèses que d'autres historiens fréquentant les mêmes archives, mais moins sensibles au " genre », n'auraient sans doute pas formulées.

L'ouvrage est muni d'une série d'illustrations reproduites en couleur par l'éditeur et sur papier glacé. On retrouve notamment de superbes plans du XVIII siècle où l'auteur sait aller chercher le moindre indice, sans pour autant extrapoler inutilement. La planche 1, par exemple, a donné le détail reproduit en couverture qui, à première vue anodin sur le plan (sans doute jamais remarqué), donne à l'auteur une occasion de discourir sur le caractère divisé entre terre et mer des " gens du rivage » qu'elle étudie. De plus, l'ouvrage fournit en annexes des transcriptions de documents inédits très intéressants, notamment l'annexe 1, une levée de cadavre de 1717, issue de sources relevant des amirautés, trop peu utilisées. Les conclusions de chapitres et la conclusion finale sont d'une clarté irréprochable. Le lecteur pressé comme l'assidu saura tirer profit de ce bel ouvrage qui éclaire le rapport au large très complexe d'une société posée entre terre et mer, humainement comme géographiquement. Une identité littorale, donc? Oui, répond $\mathrm{E}$. Charpentier, mais d'abord une appartenance au village ou à la paroisse, en somme une pluralité d'identités littorales. 